\title{
WELL-COMPOSED IMAGES AND RIGID TRANSFORMATIONS
}

\author{
Phuc Ngo $^{(1)}$, Nicolas Passat ${ }^{(2)}$, Yukiko Kenmochi(1) ${ }^{(1)}$ Hugues Talbot $^{(1)}$ \\ (1) Université Paris-Est, LIGM, ESIEE-Paris, France \\ (2) Université de Reims, CReSTIC, France
}

\begin{abstract}
We study the conditions under which the topological properties of a 2D well-composed binary image are preserved under arbitrary rigid transformations. This work initiates a more global study of digital image topological properties under such transformations, which is a crucial but under-considered problem in the context of image processing, e.g., for image registration and warping.
\end{abstract}

Index Terms - Well-composed images, rigid transformation, digital topology.

\section{INTRODUCTION}

Preservation of digital image topological properties is a crucial problem in several application fields (e.g remote sensing [2], medical imaging [1]). This problem has been considered both from the viewpoints of registration [3] and warping [4]. The majority of efforts in this direction have been devoted to deal with complex transformations, while simpler cases have been somewhat neglected. Indeed, simple transformations are generally assumed to be trivially handled, for in the continuous case (i.e., in $\mathbb{R}^{n}$ ) they are generally topology-preserving (e.g., translations or rotations). In contrast, this is not necessarily the case for more complex cases, for instance those induced by nonrigid registration [5]. Based on this "continuous" assumption, it is often thought that for simple transformations topological properties can still be easily handled in the discrete case (i.e., in $\mathbb{Z}^{n}$ ). This is in fact not the case.

In the case of discrete rigid transformations [6] (i.e. translations and rotations, followed by a digitisation), some topological issues were identified in [7]. These are directly induced by the sampling, which is mandatory to guarantee the stability of the transformations within $\mathbb{Z}^{n}$.

In this article, we propose a preliminary study that deals with necessary and sufficient conditions under which the topological properties of binary digital images in $\mathbb{Z}^{2}$ are preserved under any rigid transformations. We develop this study in the framework of well-composed images [8], by considering transformations in the Eulerian model. We show that such conditions can be checked in linear time with respect to the

This research was partially funded from the French Agence Nationale de la Recherche (Grant Agreement ANR-2010-BLAN-0205 03). size of images. A procedure for enforcing these conditions prior to transformations is also given.

The article is organised as follows. Sec. 2 provides background notions. Sec. 3 establishes the conditions for topological invariance of well-composed images under rigid transformations. Sec. 4 presents experiments and methodological remarks. Sec. 5 concludes the article by perspective works.

\section{BACKGROUND NOTIONS}

\subsection{Discrete rigid transformations}

In $\mathbb{R}^{2}$, a rigid transformation is a function $\mathcal{U}: \mathbb{R}^{2} \rightarrow \mathbb{R}^{2}$ defined for any $\boldsymbol{x}=(x, y) \in \mathbb{R}^{2}$ by

$$
\mathcal{U}(\boldsymbol{x})=\left(\begin{array}{cc}
\cos \theta & -\sin \theta \\
\sin \theta & \cos \theta
\end{array}\right)\left(\begin{array}{l}
x \\
y
\end{array}\right)+\left(\begin{array}{l}
a \\
b
\end{array}\right)
$$

where $a, b \in \mathbb{R}$ and $\theta \in[0,2 \pi)$. The function $\mathcal{U}$ is a bijection, and we note $\mathcal{T}=\mathcal{U}^{-1}$ its inverse function, which is also a rigid transformation. These definitions cannot be directly applied to the case of digital images, since there is no guarantee that $\mathcal{U}\left(\mathbb{Z}^{2}\right) \subseteq \mathbb{Z}^{2}$. The handling of discrete rigid transformations then requires to consider a discretisation operator $D: \mathbb{R}^{2} \rightarrow \mathbb{Z}^{2}$ (e.g. the standard rounding function, which is the one we use), to define discrete analogues of $\mathcal{U}$ and $\mathcal{T}$, namely $U=D \circ \mathcal{U}_{\mid \mathbb{Z}^{2}}$ and $T=D \circ \mathcal{T}_{\mid \mathbb{Z}^{2}}$.

In this work, we consider the Eulerian (backwards) transformation model. More precisely, given an image $I: \mathbb{Z}^{2} \rightarrow \mathbb{V}$ and a rigid transformation $\mathcal{U}: \mathbb{R}^{2} \rightarrow \mathbb{R}^{2}$, the transformed image $I^{\prime}: \mathbb{Z}^{2} \rightarrow \mathbb{V}$, with respect to $I$ and $\mathcal{U}$, is defined as

$$
I^{\prime}=I \circ D \circ\left(\mathcal{U}^{-1}\right)_{\mid \mathbb{Z}^{2}}=I \circ T \text {. }
$$

In the case of binary images (namely, $\mathbb{V}=\{0,1\}$ ) the image $I$ (resp. $I^{\prime}$ ) can be modeled by the set $S=\{\boldsymbol{p} \mid I(\boldsymbol{p})=1\} \subset$ $\mathbb{Z}^{2}$ (resp. $S^{\prime}=\left\{\boldsymbol{p} \mid I^{\prime}(\boldsymbol{p})=1\right\} \subset \mathbb{Z}^{2}$ ), and its complement $\bar{S}=\mathbb{Z}^{2} \backslash S$ (resp. $\overline{S^{\prime}}=\mathbb{Z}^{2} \backslash S^{\prime}$ ). Then, Eq. (2) is rewritten as

$$
\begin{aligned}
& S^{\prime}=\mathbb{Z}^{2} \cap \mathcal{U}(S \oplus \square), \\
& \overline{S^{\prime}}=\mathbb{Z}^{2} \cap \mathcal{U}(\bar{S} \oplus \square),
\end{aligned}
$$

where $\oplus$ is the dilation operator (Minkowski addition) [9] and $\square=\left[-\frac{1}{2}, \frac{1}{2}\right]^{2} \subset \mathbb{R}^{2}$ is the unit square (i.e., pixel) element. 

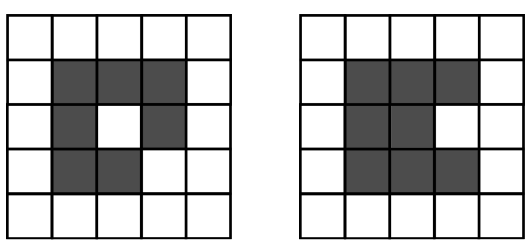

Fig. 1: Left: $S$ (in black) is weakly well-composed, while $\bar{S}$ (in white) is not. Right: both $S$ and $\bar{S}$ are well-composed.

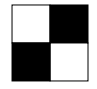

(a) $\mathscr{C}_{1}$

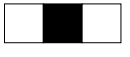

(b) $\mathscr{C}_{2}$

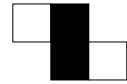

(c) $\mathscr{C}_{3}$
Fig. 2: (a) Configuration forbidden in well-composed images (see Th. 3). (a,b,c) Configurations forbidden in regular images (see Def. 9). (These configurations are given up to $\pi / 2$ rotations, symmetries and values inversion.)

Remark 1. In this work, we assume that $\mathcal{U}$ is such that $\mathbb{Z}^{2}$ does not intersect any transformed pixel border. In other words, Eqs. (2)-(4) lead to same results for $\square=\left[-\frac{1}{2}, \frac{1}{2}\right]^{2}$ or $]-\frac{1}{2}, \frac{1}{2}\left[{ }^{2}\right.$. From a theoretical viewpoint, this allows us to develop a general discussion without confusing variants related to the definition of $D$. This assumption is compliant with computer-based applications, that generally rely on floating point arithmetic.

\subsection{Digital topology and well-composed sets}

Digital topology [10] provides an efficient framework for handling the topology of binary images in $\mathbb{Z}^{n}$. It relies on a graph structure that models the adjacency relations between the points of $S$ and $\bar{S}$. To deal with topological paradoxes related to the discrete version of the Jordan theorem, a couple of dual adjacencies [11] are defined from the $L^{1}$ and $L^{\infty}$ norms, leading to the well-known 4- and 8-adjacencies in $\mathbb{Z}^{2}[10]$.

From these (irreflexive and symmetric) 4-/8-adjacency relations over the points of $\mathbb{Z}^{2}$, we obtain by reflexive/transitive closure, some equivalence relations (the 4-/8-connectedness), and their equivalence classes (the 4-/8-connected components), associated either to $S$ or $\bar{S}$. The "nested" relation between all these components can be modeled by a tree structure, called the adjacency tree [12], in which the root is the unique infinite component of the background, and each edge corresponds to the adjacency of two connected components. In particular, in $\mathbb{Z}^{2}$, two binary images with the same isomorphic adjacency trees are topologically equivalent [13].

Based on the digital topology framework, several notions of well-composition [8] have been introduced (see Fig. 1), to characterise the binary images whose structure intrinsically avoids the topological issues of the Jordan theorem.

Definition 2 (Well-composed sets [8]). We say that $S$ is weakly well-composed if any 8-connected component of $S$ is also a 4-connected component. We say that $S$ (resp. I) is well-composed if both $S$ and $\bar{S}$ are weakly well-composed.
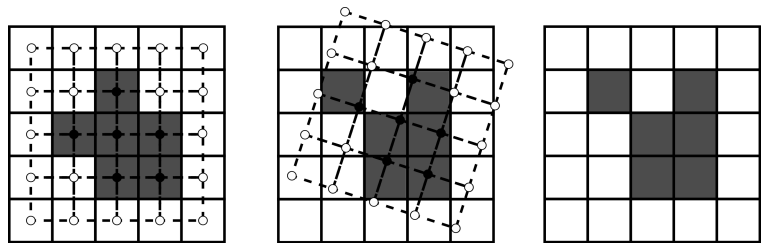

Fig. 3: Left: a well-composed image and the grid modelling its discrete structure. Middle: a rigid transformation applied on this grid. Right: the transformed image, which is not wellcomposed, and whose adjacency tree differs from the one of the initial image (with the 4 -adjacency model).

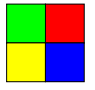

(a) $\boxplus, \mathscr{P}_{1}$

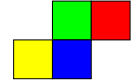

(b) $\mathscr{P}_{2}$

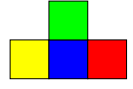

(c) $\mathscr{P}_{3}$

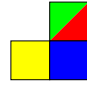

(d) $\mathscr{P}_{4}$

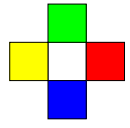

(e) $\mathscr{P}_{5}$
Fig. 4: (a) The pattern $\boxplus \subset \mathbb{Z}^{2}$. (a-e) The patterns $\mathscr{P}_{i}$ that can verify $T(\boxplus)=\mathscr{P}_{i}$ (the colours represent the point correspondance between $\boxplus$ and $\mathscr{P}_{i}$ ).(See Prop. 6.)

When interpreting digital topology in a continuous framework [14] that relies, e.g, on cubical complexes [15], a binary digital image is well-composed iff the boundaries shared by the foreground and background pixels constitute a manifold [16]. Further, it has been proved that well-composition be locally characterized by observing only the $2 \times 2$ samples of an image.

Theorem 3 ([8]). A 2D image is well-composed iff it does not contain the configuration $\mathscr{C}_{1}$ illustrated in Fig. 2(a).

\section{TOPOLOGICAL INVARIANCE UNDER RIGID TRANSFORMATIONS}

Well-composed images present nice topological properties, that may however be altered by discrete rigid transformations (see Fig. 3). We propose to investigate conditions that guarantee the topological invariance of a well-composed image.

Definition 4 (Topological invariance). We say that a wellcomposed image I is topologically invariant if any transformed image $I^{\prime}=I \circ T:(i)$ is well-composed, and (ii) has an adjacency tree isomorphic to the one of $I$.

In the following, we present several properties and lemmas which are used for proving our main result (Th. 18).

\subsection{Preliminary properties}

Let $\boxminus=\{x\} \times\{y, y+1\}$ or $\{x, x+1\} \times\{y\} \subset \mathbb{Z}^{2}$. Let $\boxplus=\{x, x+1\} \times\{y, y+1\} \subset \mathbb{Z}^{2}$. The following properties (given for any $T, \boxminus$ and $\boxplus$ ) derive from Eq. (2).

Property 5 ([17]). There exists $\boldsymbol{p} \in \mathbb{Z}^{2}$ such that $T(\boldsymbol{p}) \in \boxminus$.

Property 6 ([7]). There exist 5 patterns that can be equal to $T(\boxplus)$, up to $\pi / 2$ rotations and symmetries (see Fig. 4). 
Property 7. If $\boxplus_{1} \cap \boxplus_{2} \neq \emptyset$, then any set $P \subset \mathbb{Z}^{2}$, such that $T(P)=\boxplus_{1} \cup \boxplus_{2}$, is 4-connected.

\subsection{Necessary condition for topological invariance}

The next result is easy to prove by combinatorial analysis.

Lemma 8. If I contains $\mathscr{C}_{2}$ (resp. $\mathscr{C}_{3}$ ), then it does not satisfy condition (ii) (resp. (i)) of Def. 4.

We now introduce a notion of regularity, that strenghtens the notion of well-composition.

Definition 9 (Regularity). We say that $I$ is regular if it does not contain any of $\mathscr{C}_{1}, \mathscr{C}_{2}$ and $\mathscr{C}_{3}$.

From Defs. 4 and 9, and Lem. 8, we then derive the following necessary condition for topological invariance.

Proposition 10. If an image I is topologically invariant, then it is regular.

\subsection{Sufficient condition for well-composition}

We now show that regularity is a sufficient condition to satisfy condition $(i)$ of Def. 4.

Proposition 11. If I is a regular image, then any transformed image $I^{\prime}=I \circ T$ is well-composed.

Proof Let $T$ be such that $I^{\prime}=I \circ T$ is not well-composed. Then $I^{\prime}$ has a pattern $\boxplus$ that is a $\mathscr{C}_{1}$ (Th. 3 ), and derived from a $\mathscr{P}_{i}$, for $i=1 . .5$, in $I$ (Prop. 6). But then $\mathscr{P}_{1}$ is a $\mathscr{C}_{1}, \mathscr{P}_{2}$ is a $\mathscr{C}_{3}, \mathscr{P}_{3}$ and $\mathscr{P}_{5}$ contain a $\mathscr{C}_{2}$, and $\mathscr{P}_{4}$ is impossible. Thus, $I$ is non-regular. The result follows by contraposition.

\subsection{Regularity and 4-adjacency}

We now introduce a new adjacency relation, that strenghtens the standard 4-adjacency in $\mathbb{Z}^{2}$.

Definition 12 ( $\boxplus$-adjacency). The points $\boldsymbol{p}, \boldsymbol{q} \in S$ (resp. $\bar{S}$ ) are $\boxplus$-adjacent if $(i)$ they are 4-adjacent, and $(i i)$ there exists $\boxplus \subseteq S($ resp. $\bar{S})$ such that $\boldsymbol{p}, \boldsymbol{q} \in \boxplus$.

We then derive the notions of $\boxplus$-connectedness (equivalence relation) and $\boxplus$-connected components (equivalence classes). It is plain that the $\boxplus$-adjacency implies the 4 -adjacency. In the context of regular images, the counterpart is also true.

Lemma 13. If I is a regular image, then the 4-adjacency is equal to the $\boxplus$-adjacency in $S$ and $\bar{S}$.

Proof Let $\boldsymbol{p}, \boldsymbol{q} \in S$ be 4 -adjacent (the same holds for $\bar{S}$ ). We set $\boldsymbol{p}=(x, y)$ and $\boldsymbol{q}=(x, y+1)$ (the same holds up to $\pi / 2$ rotation). We have $\boldsymbol{r}^{\prime}=(x-1, y) \in S$ or $\boldsymbol{r}=$ $(x+1, y) \in S$, otherwise $\left\{\boldsymbol{r}^{\prime}, \boldsymbol{p}, \boldsymbol{r}\right\}$ is a $\mathscr{C}_{2}$; for instance we assume $\boldsymbol{r} \in S$. Let $\boldsymbol{s}=(x+1, y+1)$. If $s \in S$, then we have $\{\boldsymbol{p}, \boldsymbol{q}, \boldsymbol{r}, \boldsymbol{s}\}=\boxplus \in S$. If $\boldsymbol{s} \notin S$, then we have $\boldsymbol{s}^{\prime}=(x-1, y+1) \in S$, otherwise $\left\{\boldsymbol{s}^{\prime}, \boldsymbol{q}, \boldsymbol{s}\right\}$ is a $\mathscr{C}_{2}$. Thus, we have $\boldsymbol{r}^{\prime}=(x-1, y) \in S$, otherwise $\left\{\boldsymbol{r}^{\prime}, \boldsymbol{p}, \boldsymbol{q}, \boldsymbol{s}\right\}$ is a $\mathscr{C}_{3}$. Finally, we have $\left\{\boldsymbol{p}, \boldsymbol{q}, \boldsymbol{s}^{\prime}, \boldsymbol{r}^{\prime}\right\}=\boxplus \in S$.

\subsection{Sufficient condition for topological invariance}

We note $\frown S$ (resp. $\left.\frown S^{\prime}\right)$ the 4-adjacency relation on $S$ (resp. $\left.S^{\prime}\right)$, and $\sim_{S}$ (resp. $\left.\sim_{S^{\prime}}\right)$ the 4-connectedness relation on $S$ (resp. $S^{\prime}$ ). We recall that $T_{\mid S^{\prime}}$ is the restriction of $T$ to $S^{\prime}$. (Due to the symmetry of Def. 2, the results of Lem. 14, Cor. 15 and Lem. 16, stated for $S / S^{\prime}$ are also valid for $\bar{S} / \overline{S^{\prime}}$.)

Lemma 14. If $I$ is a well-composed image, then $T_{\mid S^{\prime}}$ establishes a homomorphism from $\left(S^{\prime}, \frown S^{\prime}\right)$ to $\left(S, \sim_{S}\right)$.

Proof Let $\boldsymbol{p}^{\prime} \frown S^{\prime} \boldsymbol{q}^{\prime}, \boldsymbol{p}=T_{\mid S^{\prime}}\left(\boldsymbol{p}^{\prime}\right)$ and $\boldsymbol{q}=T_{\mid S^{\prime}}\left(\boldsymbol{q}^{\prime}\right)$. From Eq. (2), we can have: (i) $\boldsymbol{p}=\boldsymbol{q}$, and then $\boldsymbol{p} \sim_{S} \boldsymbol{q}$; (ii) $\boldsymbol{p} \frown_{S} \boldsymbol{q}$, and then $\boldsymbol{p} \sim_{S} \boldsymbol{q}$; or (iii) $\boldsymbol{p}, \boldsymbol{q}$ are 8-adjacent, and thus 8-connected, and then $\boldsymbol{p} \sim_{S} \boldsymbol{q}$ is derived from Def. 2 .

Corollary 15. Let $C^{\prime} \in\left[S^{\prime}\right]_{\sim_{S^{\prime}}}$ be a 4-connected component of $S^{\prime}$. If I is well-composed, then there exists one 4-connected component $C \in[S]_{\sim_{S}}$ of $S$ such that $T_{\mid S^{\prime}}\left(C^{\prime}\right) \subseteq C$.

We can then define the function $\mathfrak{T}_{S^{\prime}}:\left[S^{\prime}\right]_{\sim_{S^{\prime}}} \rightarrow[S]_{\sim_{S}}$, such that for any $C^{\prime} \in\left[S^{\prime}\right]_{\sim_{S^{\prime}}}$ we have $T_{\mid S^{\prime}}\left(C^{\prime}\right) \subseteq \mathfrak{T}_{S^{\prime}}\left(C^{\prime}\right)$.

Lemma 16. If I is a regular image, then $\mathfrak{T}_{S^{\prime}}$ is a bijection.

Proof (Surjectivity) Let $C \in[S]_{\sim_{S}}$ and $\boldsymbol{p} \in C$. There exists $\boldsymbol{q} \in C$ such that $\boldsymbol{p} \frown S \boldsymbol{q}$, otherwise, $I$ contains $\mathscr{C}_{2}$ centered on $\boldsymbol{p}$. Then $\{\boldsymbol{p}, \boldsymbol{q}\}=\boxminus$. There exists $\boldsymbol{r} \in S^{\prime}$ such that $T_{\mid S^{\prime}}(\boldsymbol{r}) \in\{\boldsymbol{p}, \boldsymbol{q}\} \subseteq C$ (Prop. 5). Then $\mathfrak{T}_{S}$ is a surjection (Cor. 15). (Injectivity) Let $\boldsymbol{p}^{\prime}, \boldsymbol{q}^{\prime} \in S^{\prime}, \boldsymbol{p}=T_{\mid S^{\prime}}\left(\boldsymbol{p}^{\prime}\right)$ and $\boldsymbol{q}=T_{\mid S^{\prime}}\left(\boldsymbol{q}^{\prime}\right)$. Suppose that $\boldsymbol{p}, \boldsymbol{q} \in C \in[S]_{\sim_{S}}$, then $\boldsymbol{p}, \boldsymbol{q}$ are 4-connected, and therefore $\boxplus$-connected in $S$ (Lem. 13). In other words, we have $\boldsymbol{p}, \boldsymbol{q} \in \bigcup_{i=0}^{k} \boxplus_{i}(k \geq 0)$, where $\forall i \in \llbracket 0, k \rrbracket, \boxplus_{i} \subseteq S$ and $\forall i \in \llbracket 0, k-1 \rrbracket, \boxplus_{i} \cap \boxplus_{i+1} \neq \emptyset$. For any $i \in \llbracket 0, k \rrbracket$, we define $P_{i} \subset S^{\prime}$ such that $T_{\mid S^{\prime}}\left(P_{i}\right)=\boxplus_{i}$. Any $P_{i}$ is 4 -connected (Prop. 7 for $\boxplus_{1}=\boxplus_{2}=\boxplus_{i}$ ), and then $\bigcup_{i=0}^{k} P_{i}$ is also 4-connected (Props. 7). Consequently, there exists $C^{\prime} \in\left[S^{\prime}\right]_{\sim_{S^{\prime}}}$ such that $\boldsymbol{p}^{\prime}, \boldsymbol{q}^{\prime} \in \bigcup_{i=0}^{k} P_{i} \subseteq C^{\prime}$. It follows that $\mathfrak{T}_{S}$ is an injection.

Let $\mathcal{C}_{I}=[S]_{\sim_{S}} \cup[\bar{S}]_{\sim_{\bar{S}}}$ and $\mathcal{C}_{I^{\prime}}=\left[S^{\prime}\right]_{\sim_{S^{\prime}}} \cup\left[\overline{S^{\prime}}\right]_{\sim_{S^{\prime}}}$. We define the function $\mathfrak{T}_{S^{\prime}} \cup \mathfrak{T}_{\overline{S^{\prime}}}=\mathfrak{T}_{I^{\prime}}: \mathcal{C}_{I^{\prime}} \rightarrow \mathcal{C}_{I}$, which is a bijection between the 4-connected components of $I^{\prime}$ and those of $I$. We say that $C_{1}^{\prime} \neq C_{2}^{\prime} \in \mathcal{C}_{I^{\prime}}$ (resp. $C_{1} \neq C_{2} \in \mathcal{C}_{I}$ ) are adjacent, and we note $C_{1}^{\prime} \frown_{I^{\prime}} C_{2}^{\prime}$ (resp. $C_{1} \frown_{I} C_{2}$ ), if there exist $\boldsymbol{p}^{\prime} \in C_{1}^{\prime}, \boldsymbol{q}^{\prime} \in C_{2}^{\prime}$ (resp. $\boldsymbol{p} \in C_{1}, \boldsymbol{q} \in C_{2}$ ) that are 4 -adjacent. The adjacency tree [12] of $I^{\prime}$ (resp. $I$ ) is then defined by $\left(\mathcal{C}_{I^{\prime}}, \frown_{I^{\prime}}\right)$ (resp. $\left.\left(\mathcal{C}_{I}, \frown_{I}\right)\right)$.

Proposition 17. If $I$ is a regular image, then $\mathfrak{T}_{I^{\prime}}$ establishes an isomorphism between $\left(\mathcal{C}_{I^{\prime}}, \frown_{I^{\prime}}\right)$ and $\left(\mathcal{C}_{I}, \frown_{I}\right)$.

Proof Let $C_{1}^{\prime} \frown_{I^{\prime}} C_{2}^{\prime}$. Up to reindexing, we have $C_{1}^{\prime} \in$ $\left[S^{\prime}\right]_{\sim_{S^{\prime}}}, C_{2}^{\prime} \in\left[\overline{S^{\prime}}\right]_{\sim \overline{S^{\prime}}}$. Let $\boldsymbol{p}^{\prime} \in C_{1}^{\prime}, \boldsymbol{q}^{\prime} \in C_{2}^{\prime}$ be 4-adjacent, $\boldsymbol{p}=T_{\mid S^{\prime}}\left(\boldsymbol{p}^{\prime}\right) \in \mathfrak{T}_{I^{\prime}}\left(C_{1}^{\prime}\right)$ and $\boldsymbol{q}=T_{\mid \overline{S^{\prime}}}\left(\boldsymbol{q}^{\prime}\right) \in \mathfrak{T}_{I^{\prime}}\left(C_{2}^{\prime}\right)$. From Eq. (2), we can have: $(i) \boldsymbol{p}, \boldsymbol{q}$ are 4 -adjacent, and then $\mathfrak{T}_{I^{\prime}}\left(C_{1}^{\prime}\right) \frown_{I} \mathfrak{T}_{I^{\prime}}\left(C_{2}^{\prime}\right)$; or $($ ii $) \boldsymbol{p}, \boldsymbol{q}$ are 8-adjacent (but not 4adjacent). In the second case, let $\boldsymbol{r}$ be 4 -adjacent with both 
$\boldsymbol{p}$ and $\boldsymbol{q}$. We have either $\boldsymbol{r} \in \mathfrak{T}_{I^{\prime}}\left(C_{1}^{\prime}\right)$ or $\mathfrak{T}_{I^{\prime}}\left(C_{2}^{\prime}\right)$, and then $\mathfrak{T}_{I^{\prime}}\left(C_{1}^{\prime}\right) \frown_{I} \mathfrak{T}_{I^{\prime}}\left(C_{2}^{\prime}\right)$.

Let $C_{1} \frown_{I} C_{2}$. Up to reindexing, we have $C_{1} \in[S]_{\sim_{S}}$, $C_{2} \in[\bar{S}]_{\sim_{\bar{S}}}$. Let $\boldsymbol{p} \in C_{1}, \boldsymbol{q} \in C_{2}$ be 4 -adjacent. Up to $\pi / 2$ rotation, we have $\boldsymbol{p}=(x, y), \boldsymbol{q}=(x, y+1)$. Case $(i)$ : we have $\boldsymbol{r}=(x+1, y) \in C_{1}$ and $s=(x+1, y+1) \in C_{2}$. Then we have $\{\boldsymbol{p}, \boldsymbol{q}, \boldsymbol{r}, \boldsymbol{s}\}=\boxplus$ such that $\{\boldsymbol{p}, \boldsymbol{r}\}=\boxminus \subset$ $C_{1}$ and $\{\boldsymbol{q}, \boldsymbol{s}\}=\boxminus \subset C_{2}$. Let $P \subset \mathbb{Z}^{2}$ such that $T(P)=\{\boldsymbol{p}, \boldsymbol{q}, \boldsymbol{r}, \boldsymbol{s}\}$. We have $P \cap \mathfrak{T}_{I^{\prime}}^{-1}\left(C_{1}\right) \neq \emptyset$ and $P \cap \mathfrak{T}_{I^{\prime}}^{-1}\left(C_{2}\right) \neq \emptyset$ (Prop. 5). Moreover, $P$ is 4-connected (Prop. 7). Then, we have $\mathfrak{T}_{I^{\prime}}^{-1}\left(C_{1}\right) \frown_{I} \mathfrak{T}_{I^{\prime}}^{-1}\left(C_{2}\right)$. Case (ii): we have $\boldsymbol{r}, \boldsymbol{s} \in C_{1}$. Let $\boxplus_{1}=\{\boldsymbol{p}, \boldsymbol{q}, \boldsymbol{r}, \boldsymbol{s}\}$ and $\boxplus_{2}=\boldsymbol{q}+\{-1,0\} \times\{0,1\}$. We have $\boxplus_{2} \subseteq C_{2}$ (by a similar reasoning to that for Lem. 13). Let $P \subset \mathbb{Z}^{2}$ such that $T(P)=\boxplus_{1} \cup \boxplus_{2}$. We have $P \cap \mathfrak{T}_{I^{\prime}}^{-1}\left(C_{1}\right) \neq \emptyset$ and $P \cap \mathfrak{T}_{I^{\prime}}^{-1}\left(C_{2}\right) \neq \emptyset$ (Prop. 5). Moreover, $P$ is 4-connected (Prop. 7). Then, we have $\mathfrak{T}_{I^{\prime}}^{-1}\left(C_{1}\right) \frown_{I} \mathfrak{T}_{I^{\prime}}^{-1}\left(C_{2}\right)$. The other cases are impossible or equivalent to the above two cases, by symmetry between $S$ and $\bar{S}$.

Combining properties 10, 11 and 17, we obtain our main result.

Theorem 18. The image $I: \mathbb{Z}^{2} \rightarrow\{0,1\}$ is topologically invariant iff it is regular.

This result enables us, in particular, to check the topological invariance of an image $I$ by simply scanning it once to identify potential occurrences of $\mathscr{C}_{1}, \mathscr{C}_{2}$ and $\mathscr{C}_{3}$.

\section{EXPERIMENTS AND METHODOLOGY}

From Sec. 3, we can determine in linear time whether a 2D binary well-composed image is topologically invariant using the introduced notion of regularity. The analytic usefulness of these results is illustrated in Fig. 5(a-d).

As well as allowing us to analyse images, these results also lead to solutions for improving their topological robustness. Indeed, once the pixels that forbid topological invariance have been identified (see Fig. 5(c)), images can be processed to locally modify the corresponding areas, in order to fulfill the regularity requirements (see Fig. 5(e,f)). This process must be performed iteratively until convergence, and can still fail in some cases, e.g., when the images present a fine texture (see Fig. 6).

In such cases, a super-resolution strategy can be considered. Indeed, by doubling the resolution, i.e., by associating a $2 \times 2$ pixel square to each initial pixel, a well-composed image necessarily becomes regular. Moreover, this strategy can be associated to other super-resolution paradigms based, e.g., on Khalimsky grids [18], to transform any $(4,8)$ or $(8,4)$ adjacency binary image into a well-composed, and finally regular one.

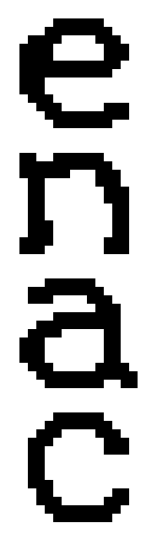

(a)
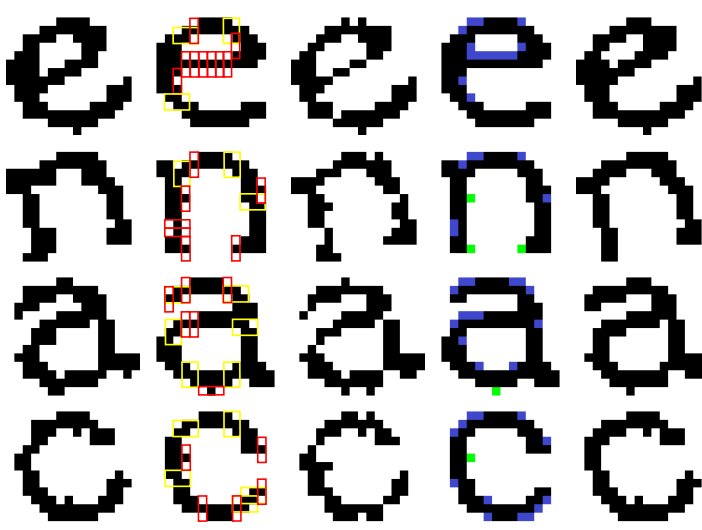

(b)

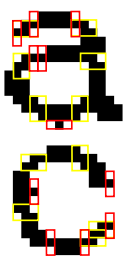

(c)

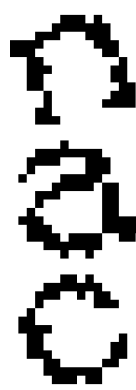

(d)

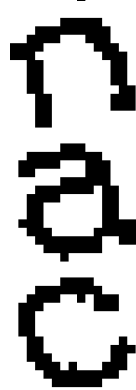

(f)

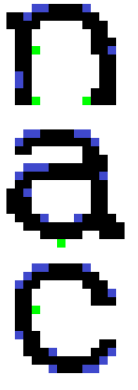

(e)
Fig. 5: (a) Regular images. (b) Rigid transformations of (a), with topological preservation. (c) Well-composed but nonregular images (the occurrences of critical configurations $\mathscr{C}_{2}$ and $\mathscr{C}_{3}$ are depicted by red and yellow frames, respectively). (d) Rigid transformations of (c), with topological alterations. (e) Corrected (regular) images obtained from (c) (in blue: added pixels; in green: removed pixels). (f) Rigid transformations of (e), with topological preservation.
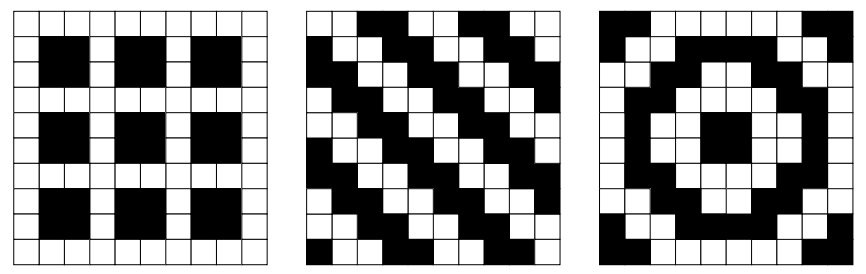

Fig. 6: Well-composed images that cannot be modified to fulfill regularity requirements without resorting to superresolution.

\section{CONCLUSION}

We have introduced the notion of regularity as a necessary and sufficient condition to ensure that the topological properties of a 2D well-composed binary image are preserved under arbitrary rigid transformation. We have proposed solutions for detecting and favoring such preservation in applications.

From a methodological viewpoint, this preliminary study opens the way to extensions in terms of dimensions (from 2D to 3D [19]), value spaces (from binary to grey-scale [20] and colour [21]), with applicative perspectives in image processing (registration, image warping, etc.). In addition, the standard digital topology case of dual $(8,4)$ - and $(4,8)$ adjacencies will also be investigated.

From a theoretical viewpoint, the relations that may exist between these results and various (continuous and/or discrete) geometrical and topological concepts will also be explored. In particular, some links may be found with notions such as the $r$-regularity [22,23] and connectivity classes [24, 25]. 


\section{REFERENCES}

[1] D. L. Pham, P.-L. Bazin, and J. L. Prince, "Digital topology in brain imaging," IEEE Signal Processing Magazine, vol. 27, no. 4, pp. 51-59, 2010.

[2] P. Soille and M. Pesaresi, "Advances in mathematical morphology applied to geoscience and remote sensing," IEEE Transactions on Geoscience and Remote Sensing, vol. 40, no. 9, pp. 2042-2055, 2002.

[3] P.-L. Bazin, L. M. Ellingsen, and D. L. Pham, "Digital homeomorphisms in deformable registration," in Information Processing in Medical Imaging (IPMI). 2007, vol. 4584 of $L N C S$, pp. 211-222, Springer.

[4] S. Faisan, N. Passat, V. Noblet, R. Chabrier, and C. Meyer, "Topology-preserving warping of binary images according to one-to-one mappings," IEEE Transactions on Image Processing, vol. 20, no. 8, pp. 21352145, 2011.

[5] V. Noblet, C. Heinrich, F. Heitz, and J.-P. Armspach, "3$\mathrm{D}$ deformable image registration: A topology preservation scheme based on hierarchical deformation models and interval analysis optimization," IEEE Transactions on Image Processing, vol. 14, no. 5, pp. 553-566, 2005.

[6] P. Ngo, Y. Kenmochi, N. Passat, and H. Talbot, "Combinatorial structure for rigid transformations in 2D digital images," Computer Vision and Image Understanding, In Press, DOI: 10.1016/j.cviu.2012.08.014.

[7] P. Ngo, Y. Kenmochi, N. Passat, and H. Talbot, "Sufficient conditions for topological invariance of 2D digital images under rigid transformations," in Discrete Geometry for Computer Imagery (DGCI). 2013, LNCS, Springer. In Press.

[8] L. J. Latecki, U. Eckhardt, and A. Rosenfeld, "Wellcomposed sets," Computer Vision and Image Understanding, vol. 61, no. 1, pp. 70-83, 1995.

[9] H. J. A. M. Heijmans and C. Ronse, "The algebraic basis of mathematical morphology. I. Dilations and erosions," Computer Vision, Graphics, and Image Processing, vol. 50, no. 3, pp. 245-295, 1990.

[10] T. Y. Kong and A. Rosenfeld, "Digital topology: Introduction and survey," Computer Vision, Graphics, and Image Processing, vol. 48, no. 3, pp. 357-393, 1989.

[11] A. Rosenfeld, "Digital topology," The American Mathematical Monthly, vol. 86, no. 8, pp. 621-630, 1979.

[12] A. Rosenfeld, "Adjacency in digital pictures," Information and Control, vol. 26, no. 1, pp. 24-33, 1974.
[13] A. Rosenfeld, T. Y. Kong, and A. Nakamura, "Topology-preserving deformations of two-valued digital pictures," Graphical Models and Image Processing, vol. 60, no. 1, pp. 24-34, 1998.

[14] L. Mazo, N. Passat, M. Couprie, and C. Ronse, "Digital imaging: A unified topological framework," Journal of Mathematical Imaging and Vision, vol. 44, no. 1, pp. 19-37, 2012.

[15] V. A. Kovalevsky, "Finite topology as applied to image analysis," Computer Vision, Graphics, and Image Processing, vol. 46, no. 2, pp. 141-161, 1989.

[16] G. Damiand, A. Dupas, and J.-O. Lachaud, "Fully deformable 3D digital partition model with topological control," Pattern Recognition Letters, vol. 32, no. 9, pp. 1374-1383, 2011.

[17] B. Nouvel and E. Rémila, "Configurations induced by discrete rotations: Periodicity and quasi-periodicity properties," Discrete Applied Mathematics, vol. 147, no. 2-3, pp. 325-343, 2005.

[18] E. Khalimsky, “Topological structures in computer science," Journal of Applied Mathematics and Simulation, vol. 1, no. 1, pp. 25-40, 1987.

[19] L. J. Latecki, “3D well-composed pictures,” Computer Vision, Graphics, and Image Processing, vol. 59, no. 3, pp. 164-172, 1997.

[20] J. Marchadier, D. Arquès, and S. Michelin, "Thinning grayscale well-composed images," Pattern Recognition Letters, vol. 25, no. 5, pp. 581-590, 2004.

[21] L. J. Latecki, "Multicolor well-composed pictures," Pattern Recognition Letters, vol. 16, no. 4, pp. 425-431, 1995.

[22] A. Gross and L. Latecki, "Digitizations preserving topological and differential geometric properties," Computer Vision and Image Understanding, vol. 62, no. 3, pp. 370-381, 1995.

[23] L. J. Latecki, C. Conrad, and A. Gross, "Preserving topology by a digitization process," Journal of Mathematical Imaging and Vision, vol. 8, no. 2, pp. 131-159, 1998.

[24] C. Ronse, "Set-theoretical algebraic approaches to connectivity in continuous or digital spaces," Journal of Mathematical Imaging and Vision, vol. 8, no. 1, pp. 4158, 1998.

[25] J. Serra, "Connectivity on complete lattices," Journal of Mathematical Imaging and Vision, vol. 9, no. 3, pp. 231-251, 1998. 